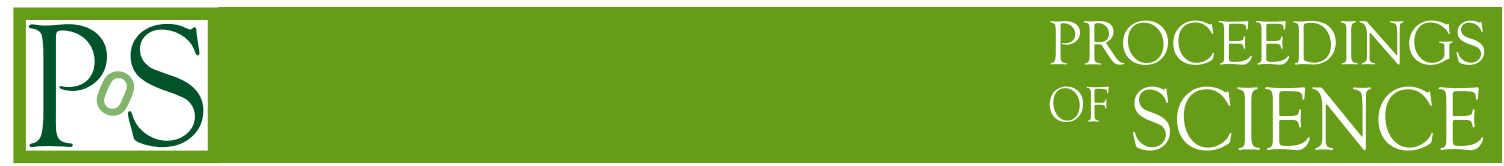

\title{
COMPASS results on hard exclusive muoproduction
}

\author{
Andrzej Sandacz* \\ (on behalf of the COMPASS Collaboration) \\ National Centre for Nuclear Research (NCBJ), Warsaw \\ E-mail: Andrzej.Sandacz@ncbj.gov.pl
}

\begin{abstract}
The high energy polarised muon beam available at CERN, with positive or negative charge, makes COMPASS a unique place for studies of General Parton Distributions (GPDs). The first GPD related COMPASS results were obtained for exclusive vector meson production on transversely polarised protons and deuterons. The data were taken in 2003-2010 with solid-state polarised targets, although without recoil detector. Results on various transverse target spin dependent azimuthal asymmetries are presented and their relation to GPDs is discussed. The dedicated COMPASS GPD program started in 2012 with commissioning of a new long liquid hydrogen target and new detectors, such as the large recoil proton detector CAMERA and the large-angle electromagnetic calorimeter. It was followed by a short pilot 'DVCS run'. The performance of the setup and first results on DVCS channel are presented. The full data taking for the GPD program approved within COMPASS-II proposal is planned in 2016 and 2017.
\end{abstract}

QCD Evolution 2016

May 30-June 03, 2016

National Institute for Subatomic Physics (Nikhef), Amsterdam

${ }^{*}$ Supported by the Polish NCN Grant 2015/18/M/ST2/00550 


\section{Introduction}

Interest in studying exclusive processes in hard scattering of leptons off nucleons has increased recently as this allows access to the Generalised Parton Distributions (GPDs) [1, 2, 3]. The GPDs contain a wealth of information on the partonic structure of the nucleon, which is the one of central problems in hadron physics. In particular, GPDs allow a novel description of the nucleon as an extended object, sometimes referred to as 3-dimensional 'nucleon tomography' [4]. GPDs also allow access to such a fundamental property of the nucleon as the orbital angular momentum of quarks [2]. For reviews of the GPDs see Refs [5, 6, 7]. The mapping of the nucleon GPDs requires comprehensive experimental studies of hard processes such as Deeply Virtual Compton Scattering (DVCS) and Hard Exclusive Meson Production (HEMP) in a broad kinematic range.

In this paper we discuss COMPASS results on exclusive vector meson production on transversely polarised protons. They provide constrains on the 'elusive' GPDs $E$, which together with the better known GPDs $H$ are related to the total angular momentum carried by partons in the nucleon [2]. We also present the preliminary result on the $t$-slope of the differential cross section for DVCS that was obtained from a short three-week data taking period in 2012 with a liquid hydrogen target and the large recoil proton detector. This first 'direct' measurement of the slope at intermediate values of $x_{B j}$ has an impact for studies of 'nucleon tomography'.

\section{Exclusive $\rho^{0}$ and $\omega$ muoproduction on transversely polarised protons}

The COMPASS collaboration has analysed exclusive vector meson production on polarised ${ }^{6} \mathrm{LiD}$ (deuterons) and $\mathrm{NH}_{3}$ (protons) targets using the data from 2003-2010. Here the results for the data taken with polarised protons will be discussed. Although no recoil proton detector was included in the used experimental setup, which is a disadvantage for measurements of exclusive processes, the analysis of these data allows us to obtain first valuable results that are sensitive to GPDs $E$ and chiral-odd GPDs.

\subsection{Experimental setup and event selection}

COMPASS is a fixed-target experiment situated at the high-intensity M2 beam-line of the CERN SPS. A detailed description of the experimental setup can be found in Ref. [8].

The $\mu^{+}$beam had a nominal momentum of $160 \mathrm{GeV} / c$ with a spread of $5 \%$ and a longitudinal polarisation of $P_{\ell} \approx-0.8$. The data were taken at a mean intensity of $3.5 \cdot 10^{8} \mu /$ spill, for a spill length of about $10 \mathrm{~s}$ every $40 \mathrm{~s}$. A measurement of the trajectory and the momentum of each incoming muon is performed upstream of the target. The momentum of the beam muon is measured with a relative precision better than $1 \%$.

The beam traverses a solid-state ammonia $\left(\mathrm{NH}_{3}\right)$ target that provides transversely polarised protons. The target is situated within a large aperture magnet with a dipole holding field of $0.5 \mathrm{~T}$. The $2.5 \mathrm{~T}$ solenoidal field is only used when polarising the target material. A mixture of liquid ${ }^{3} \mathrm{He}$ and ${ }^{4} \mathrm{He}$ is used to cool the target to $50 \mathrm{mK}$. The ammonia is contained in three cylindrical target cells with a diameter of $4 \mathrm{~cm}$, placed one after another along the beam. The central cell is $60 \mathrm{~cm}$ long and the two outer ones are $30 \mathrm{~cm}$ long, with $5 \mathrm{~cm}$ space between cells. The spin directions in 
neighbouring cells are opposite. Systematic effects due to acceptance are reduced by reversing the spin directions on a weekly basis.

The COMPASS two-stage spectrometer is designed to reconstruct scattered muons and produced hadrons in wide momentum and angular ranges. Each stage has a dipole magnet with tracking detectors before and after the magnet, hadron and electromagnetic calorimeters and muon identification. Identification of charged tracks with a RICH detector in the first stage is not used for the results presented here.

To determine the transverse target spin asymmetries for exclusive $\rho^{0}$ production the data taken in 2007 and 2010 with polarised protons are used. The details of selection of the sample are given in Ref. [9]. The essential steps of event selection and asymmetry extraction are summarised in the following. The considered events are characterised by an incoming and a scattered muon and two oppositely charged hadrons, $h^{+} h^{-}$, with all four tracks associated to a common vertex in the polarised target. In order to select events in the deep inelastic scattering regime and suppress radiative corrections, the following cuts are used: $Q^{2}>1(\mathrm{GeV} / c)^{2}, 0.003<x_{\mathrm{Bj}}<0.35, W>5$ $\mathrm{GeV}$ and $0.1<y<0.9$. The production of $\rho^{0}$ mesons is selected in the two-hadron invariant mass range $0.5 \mathrm{GeV} / c^{2}<M_{\pi^{+} \pi^{-}}<1.1 \mathrm{GeV} / c^{2}$, where for each hadron the pion mass hypothesis is assigned. This cut is optimised towards high yield and purity of $\rho^{0}$ production, as compared to nonresonant $\pi^{+} \pi^{-}$production. The measurements are performed without detection of the recoiling proton in the final state. Exclusive events are selected by choosing a range in missing energy,

$$
E_{\text {miss }}=\frac{(p+q-v)^{2}-p^{2}}{2 M_{\mathrm{p}}} .
$$

The four-momenta of proton, photon, and meson, are denoted by $p, q$, and $v$ respectively, and $M_{\mathrm{p}}$ is the proton mass. Although for exclusive events $E_{\mathrm{miss}} \approx 0$ holds, the finite experimental resolution is taken into account by selecting events in the 'signal' range $\left|E_{\text {miss }}\right|<2.5 \mathrm{GeV}$, which corresponds to $0 \pm 2 \sigma$ where $\sigma$ is the width of the Gaussian signal peak. Non-exclusive background is further suppressed by cuts on the squared transverse momentum of the vector meson with respect to the virtual-photon direction, $p_{\mathrm{T}}^{2}<0.5(\mathrm{GeV} / \mathrm{c})^{2}$, the energy of the $\rho^{0}$ in the laboratory system, $E_{\rho^{0}}>15 \mathrm{GeV}$, and the photon virtuality, $Q^{2}<10(\mathrm{GeV} / c)^{2}$. An additional cut $p_{\mathrm{T}}^{2}>0.05(\mathrm{GeV} / c)^{2}$ is used to reduce the contribution of events from coherent production on the target nuclei. The average values of the kinematic variables are $\left\langle Q^{2}\right\rangle=2.15(\mathrm{GeV} / c)^{2},\left\langle x_{\mathrm{Bj}}\right\rangle=0.039,\langle y\rangle=0.24$, $\langle W\rangle=8.13 \mathrm{GeV}$, and $\left\langle p_{\mathrm{T}}^{2}\right\rangle=0.18(\mathrm{GeV} / \mathrm{c})^{2}$. Details on the method to correct for the contribution of the semi-inclusive background in the sample and on the extraction of asymmetries are given in Ref. [10].

The analysis of exclusive $\omega$ production was performed using the data taken in 2010 with transversely polarised protons. An event to be accepted for further analysis was required to have an incident muon track, a scattered muon track, exactly two additional tracks of oppositely charged hadrons, all associated to a vertex in the polarised target material, and a single $\pi^{0}$ meson that is reconstructed using its two decay photons detected in the electromagnetic calorimeters. The photon clusters in the calorimeters have to be time-correlated with the beam track.

Most of the selections of events from exclusive $\omega$ production follow those for $\rho^{0}$ analysis, except these listed in the following. In order to select $\pi^{0}$ meson, a cut on the accepted range of invariant mass of two photons, $M_{\gamma \gamma}$, was applied, which took into account the energy-dependent 
resolution of photon energy measurements. The $\omega$ resonance was selected by the cut on the invariant mass of $\pi^{+} \pi^{-} \pi^{0}$ system, $\left|M_{\pi \pi \pi}-M_{\omega}^{\mathrm{PDG}}\right|<70 \mathrm{MeV} / c^{2}$, where $M_{\omega}^{\mathrm{PDG}}=782.65 \mathrm{MeV} / c^{2}$. Due to an additional contribution of calorimetric measurements to the experimental resolution, the exclusivity selection was changed to $\left|E_{\text {miss }}\right|<3.0 \mathrm{GeV}$. In the analysis of $\omega$ meson the signal and background asymmetries were extracted simultaneously using the unbinned maximum likelihood method. The average values of the kinematic variables are $\left\langle Q^{2}\right\rangle=2.2(\mathrm{GeV} / c)^{2},\left\langle x_{\mathrm{Bj}}\right\rangle=0.049$, $\langle y\rangle=0.18,\langle W\rangle=7.1 \mathrm{GeV}$, and $\left\langle p_{\mathrm{T}}^{2}\right\rangle=0.17(\mathrm{GeV} / c)^{2}$.

\subsection{Results and discussion}

For a transversely polarised target five single (UT) and three double (LT) spin asymmetries can be defined. These are $A_{\mathrm{UT}}^{\sin \left(\phi-\phi_{s}\right)}, A_{\mathrm{UT}}^{\sin \left(\phi+\phi_{s}\right)}, A_{\mathrm{UT}}^{\sin \left(3 \phi-\phi_{s}\right)}, A_{\mathrm{UT}}^{\sin \phi_{s}}, A_{\mathrm{UT}}^{\sin \left(2 \phi-\phi_{s}\right)}, A_{\mathrm{LT}}^{\cos \left(\phi-\phi_{s}\right)}, A_{\mathrm{LT}}^{\cos \phi_{s}}$, $A_{\mathrm{LT}}^{\cos \left(2 \phi-\phi_{s}\right)}$. Each asymmetry is related to a modulation of the cross section as a function of $\phi$ and $\phi_{s}$ angles, which is indicated by the superscript. The angle $\phi$ is the azimuthal angle between the lepton plane, given by the momenta of the incoming and the scattered leptons, and the hadron plane, given by the momenta of the virtual photon and the meson. The angle $\phi_{s}$ is the azimuthal angle between the lepton plane and the spin direction of the target nucleon. Average values of measured asymmetries for exclusive $\rho^{0}$ production are shown in Fig. 1 (left). The asymmetry $A_{\mathrm{UT}}^{\sin \phi_{S}}$ was found to be $-0.019 \pm 0.008$ (stat.) \pm 0.003 (syst.). All other asymmetries were also found to be of small magnitude but consistent with zero within experimental uncertainties. The single transverse asymmetries measured as functions of $Q^{2}, x_{\mathrm{Bj}}$ and $p_{\mathrm{T}}^{2}$ are presented in Fig. 1 (right) and compared to the predictions of the GPD-model by Goloskokov and Kroll [11].

The measured asymmetries are proportional to bilinear combinations of the helicity amplitudes $\mathcal{M}$ for the photoproduction subprocess, $A \propto \sum \mathcal{M}_{i^{\prime} m^{\prime}, i m}^{*} \mathcal{M}_{i^{\prime} m^{\prime}, j n}$, where the helicity of the virtual photon is denoted by $i, j=-1,0,+1$ and the helicity of the initial-state proton by $m, n=-\frac{1}{2},+\frac{1}{2}$. The sum runs over all combinations of spins given by the spin of the meson $i^{\prime}=-1,0,+1$ and the spin of the final-state proton $m^{\prime}=-\frac{1}{2},+\frac{1}{2}$. In the following the helicities will be labelled by only their sign or zero.

For an interpretation of results in the framework of the model, the following asymmetries are particularly interesting, for which the dependence on the helicity amplitudes reads

$$
\begin{aligned}
& \sigma_{0} A_{\mathrm{UT}}^{\sin \left(\phi-\phi_{s}\right)}=-2 \operatorname{Im}\left[\epsilon \mathcal{M}_{0-, 0+}^{*} \mathcal{M}_{0+, 0+}+\mathcal{M}_{+-,++}^{*} \mathcal{M}_{++,++}+\frac{1}{2} \mathcal{M}_{0-,++}^{*} \mathcal{M}_{0+,++}\right], \\
& \sigma_{0} A_{\mathrm{UT}}^{\sin \left(2 \phi-\phi_{s}\right)}=-\operatorname{Im}\left[\mathcal{M}_{0+,++}^{*} \mathcal{M}_{0-, 0+}\right] \\
& \sigma_{0} A_{\mathrm{UT}}^{\sin \phi_{s}}=-\operatorname{Im}\left[\mathcal{M}_{0-,++}^{*} \mathcal{M}_{0+, 0+}-\mathcal{M}_{0+,++}^{*} \mathcal{M}_{0-, 0+}\right] .
\end{aligned}
$$

Here $\sigma_{0}$ is the total unpolarised cross section, given by the sum of cross sections for longitudinally, $\sigma_{L}$, and transversely, $\sigma_{T}$, polarised virtual photons,

$$
\sigma_{0}=\frac{1}{2}\left(\sigma_{++}^{++}+\sigma_{++}^{--}\right)+\epsilon \sigma_{00}^{++}=\sigma_{T}+\epsilon \sigma_{L},
$$

and $\epsilon$ is the virtual photon polarisation parameter.

The dominant $\gamma_{L}^{*} \rightarrow \rho_{L}^{0}$ transitions are described by helicity amplitudes $\mathcal{M}_{0+, 0+}$ and $\mathcal{M}_{0-, 0+}$, which are related to chiral-even GPDs $H$ and $E$, respectively. The subscripts $L$ and $T$ denote the photon and meson helicities 0 and \pm 1 , respectively. The suppressed $\gamma_{T}^{*} \rightarrow \rho_{T}^{0}$ transitions are 


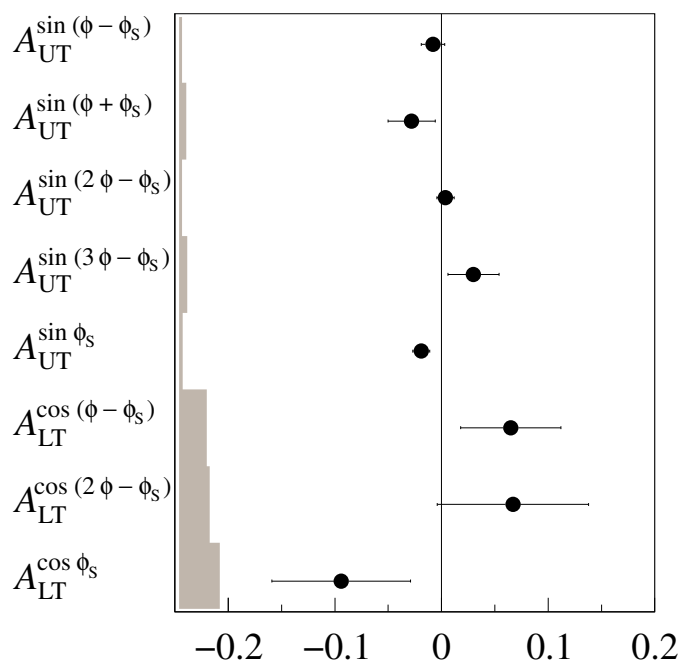

$\langle A\rangle$

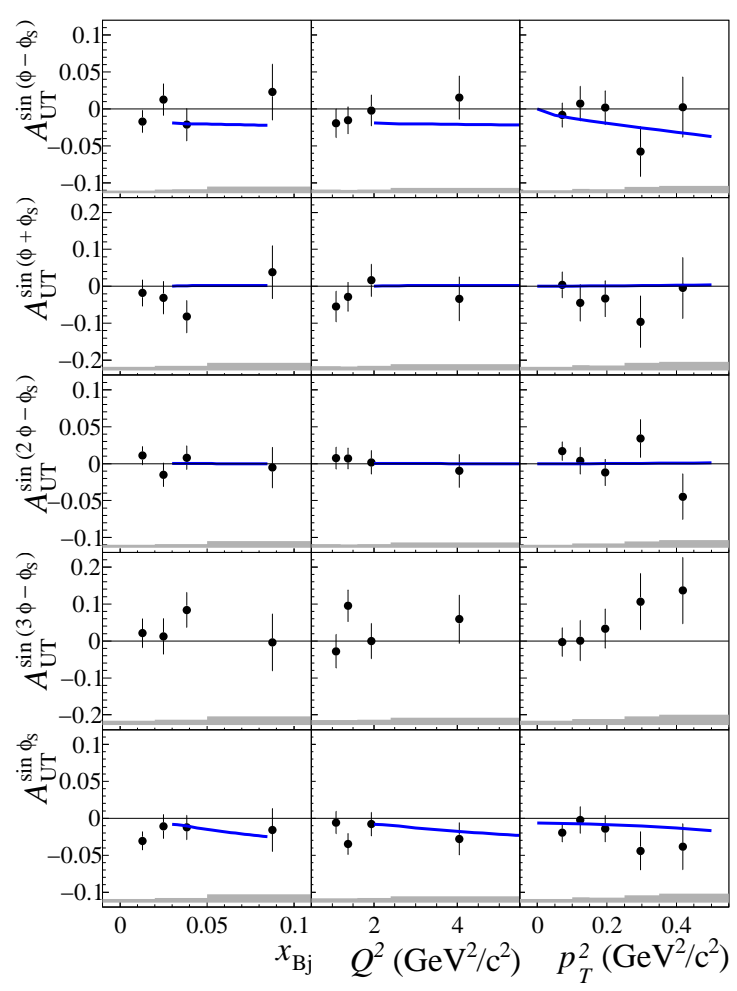

Figure 1: Left: Mean values $\langle A\rangle$ for all transverse target asymmetries for exclusive $\rho^{0}$ production. The errors bars (left bands) represent the statistical (systematic) uncertainties. Right: Single transverse target spin asymmetries for exclusive $\rho^{0}$ production. The curves show the predictions of the GPD model of Ref. [11] for the average $Q^{2}, x_{B j}$ and $p_{\mathrm{T}}^{2}$ values of the COMPASS data.

described by the helicity amplitudes $\mathcal{M}_{++,++}$and $\mathcal{M}_{+-,++}$, which are likewise related to $H$ and $E$. These GPDs are used since several years to describe DVCS and exclusie vector meson production data. By the recent inclusion of transverse, i.e. chiral-odd GPDs, it became possible to also describe $\gamma_{T}^{*} \rightarrow \rho_{L}^{0}$ transitions [11]. In their description appear the amplitudes $\mathcal{M}_{0-,++}$ related to chiral-odd GPDs $H_{T}$ and $\mathcal{M}_{0+,++}$ related to chiral-odd GPDs $\bar{E}_{T}$.

The small value of $A_{\mathrm{UT}}^{\sin \left(\phi-\phi_{s}\right)}$ asymmetry for $\rho^{0}$ is explained as an approximate cancellation of contributions from GPDs $E^{u}$ and $E^{d}$ for valence quarks $u$ and $d$, respectively, which have opposite signs but similar absolute values. The $A_{\mathrm{UT}}^{\sin \phi_{s}}$ asymmetry represents an imaginary part of two bilinear products of helicity amplitudes. The first product is related to GPDs $H$ and $H_{T}$, while the second one is related to GPDs $E$ and $\bar{E}_{T}$. The asymmetry $A_{\mathrm{UT}}^{\sin \left(2 \phi-\phi_{S}\right)}$ represents the same combination of GPDs $E$ and $\bar{E}_{T}$ as the second term in $A_{\mathrm{UT}}^{\sin \phi_{S}}$. The observation of a vanishing value for $A_{\mathrm{UT}}^{\sin \left(2 \phi-\phi_{S}\right)}$ implies that the non-vanishing value of $A_{\mathrm{UT}}^{\sin \phi_{S}}$ constitutes the first experimental evidence from hard exclusive $\rho^{0}$ leptoproduction for the existence of transverse GPDs $H_{T}$.

The preliminary result on average values of all eight transverse target asymmetries for exclusive $\omega$ production are shown in Fig. 2 (left), while the single spin asymmetries as functions of $Q^{2}$, $x_{\mathrm{Bj}}$ and $p_{\mathrm{T}}^{2}$ are presented in Fig. 2 (right) and compared to the predictions of Goloskokov-Kroll model [12]. The predictions are given for three versions of the model: with the pion-pole contribution using a positive or negative $\pi \omega$ transition form factor, and without the pion-pole contribution. 

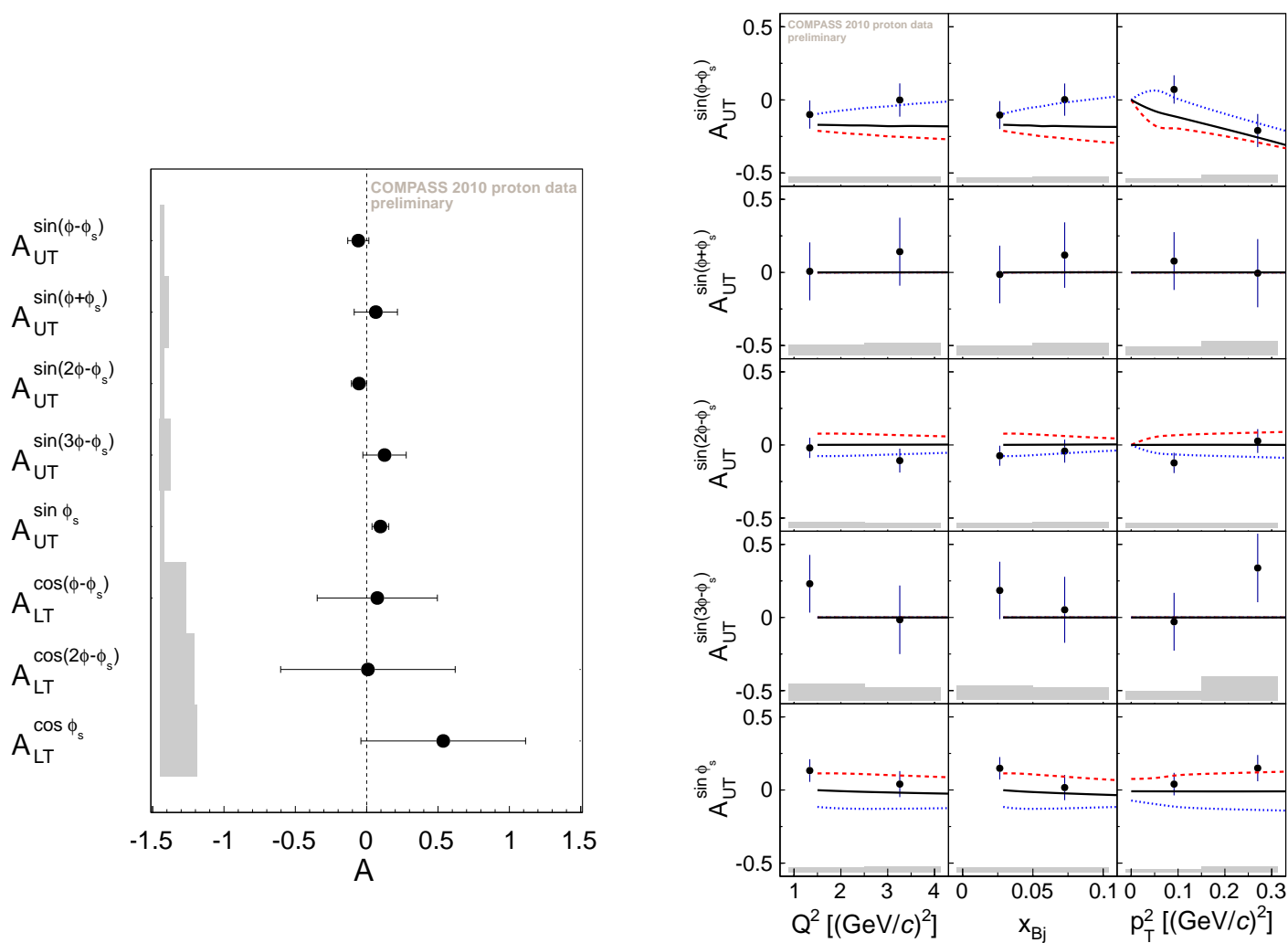

Figure 2: Left: Mean values $A$ for all transverse target asymmetries for exclusive $\omega$ production. The errors bars (left bands) represent the statistical (systematic) uncertainties. Right: Single transverse target spin asymmetries for exclusive $\omega$ production. The curves show the predictions of the GPD model of Ref. [12] for the average $Q^{2}, x_{B j}$ and $p_{\mathrm{T}}^{2}$ values of the COMPASS data. The dashed red and dotted blue lines represent the predictions with the positive and negative $\pi \omega$ form factors, respectively, while the solid black lines represent the predictions without the pion pole exchange.

The asymmetry $A_{\mathrm{UT}}^{\sin \left(\phi-\phi_{s}\right)}$ for exclusive $\omega$ production predicted by the model without pionpole contribution is -0.11 . This value is significantly different from that for exclusive $\rho^{0}$ production, which amounts to -0.01 . Thus in principle a combined analysis of results for this asymmetry for both mesons could allow a separation of the contributions of GPDs $E^{u}$ and $E^{d}$, which are different in both cases.

However, the interpretation of $\omega$ results in the context of the GPD formalism is more challenging than that for $\rho^{0}$, as exclusive $\omega$ meson production is significantly influenced by the pion-pole exchange contribution, and at present the sign of $\pi \omega$ transition form factor is unknown. By comparing the COMPASS results with the calculations of the GK model (see Fig. 2 (right)), one finds that the asymmetries $A_{\mathrm{UT}}^{\sin \left(\phi-\phi_{s}\right)}$ and $A_{\mathrm{UT}}^{\sin \left(2 \phi-\phi_{s}\right)}$ prefer the negative $\pi \omega$ transition form factor, while the asymmetry $A_{\mathrm{UT}}^{\sin \phi_{s}}$ prefers the positive one. The other measured asymmetries are not sensitive to the sign of the $\pi \omega$ form factor.

The single-spin azimuthal asymmetries for $\omega$ production on transversely polarised protons were measured also by the HERMES collaboration [13]. A direct comparison of published asymmetry values measured in both experiments in not straightforward, because definitions of physics 


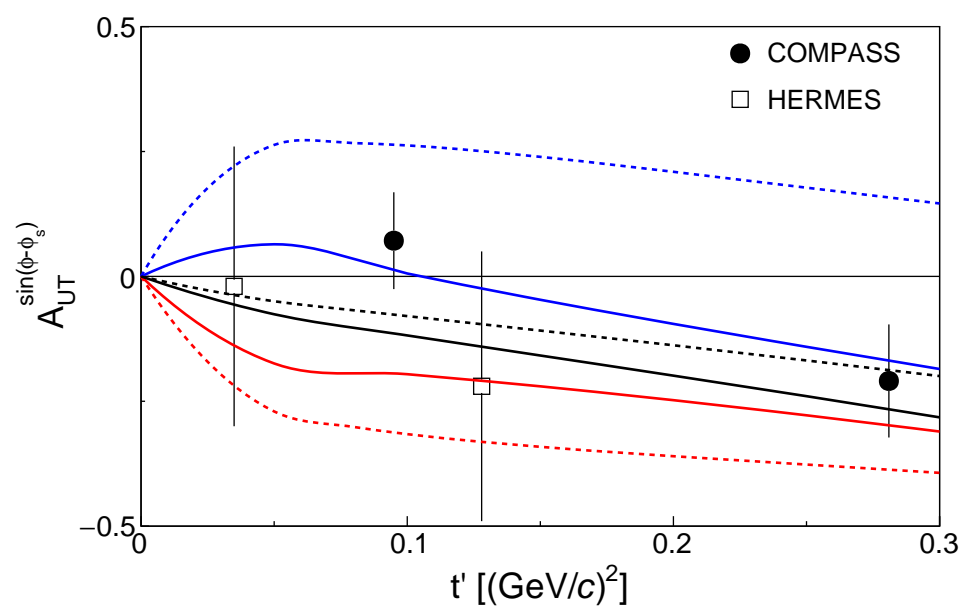

Figure 3: The asymmetry $A_{\mathrm{UT}}^{\sin \left(\phi-\phi_{s}\right)}$ for exclusive $\omega$ muoproduction measured by COMPASS (filled circles) and HERMES [13] (open squares) collaborations as a function of $t^{\prime}$. The curves show the predictions of the GPD-based model [12] given for the average $Q^{2}$ and $W$ values of the COMPASS (solid lines) and HERMES (dashed lines) data. For each set of curves, the upper (blue) and lower (red) ones are for the negative and positive $\pi \omega$ form factors, respectively, while the middle (black) one represents the predictions without the pion pole.

asymmetries are different. Such comparison is only possible for the asymmetry $A_{\mathrm{UT}}^{\sin \left(\phi-\phi_{s}\right)}$. The results from both experiments are shown as a function of $t^{\prime}$ in Fig. 3 indicating their compatibility within experimental uncertainties. Note that the COMPASS results cover a wider kinematic range and they have smaller uncertainties, for example for the asymmetry $A_{\mathrm{UT}}^{\sin \left(\phi-\phi_{s}\right)}$ by a factor larger than two.

\section{The GPD program of COMPASS-II}

The GPD part of COMPASS-II proposal [14] is devoted to measurements of both DVCS and HEMP with polarised $\mu^{+}$and $\mu^{-}$beams and a liquid hydrogen target. The new detectors, the $4 \mathrm{~m}-$ long recoil proton detector CAMERA and (a central part of) the new large-angle electromagnetic calorimeter ECAL0, which are essential for measurements of exclusive processes, were constructed and incorporated into the COMPASS setup in 2012. The commissioning of these new detectors was done in 2012 and was followed by a short DVCS pilot run. The dedicated data taking for the GPD program, with the complete calorimeter ECAL0, is foreseen in 2016-2017 for a total period of 280 days.

The recoil proton detection is based on the ToF measurement between two barrels of 24 scintillator slats read out at both ends. By installing the CAMERA around the new $2.5 \mathrm{~m}$ long $\mathrm{LH}_{2}$ target and the calorimeter ECAL0 the COMPASS has been converted into a facility measuring exclusive reactions within a kinematic domain from $x_{\mathrm{Bj}} \sim 0.01$ to $\sim 0.1$, which cannot be explored at any other existing or planned facility in the near future.

An efficient selection of exclusive events and suppression of the background was possible by using the combined information from the forward COMPASS detectors and the CAMERA. As an example a result for exclusive single photon production, obtained from the 2012 DVCS pilot run, is presented in the following. The selection of events is discussed in detail in Ref. [15]. 

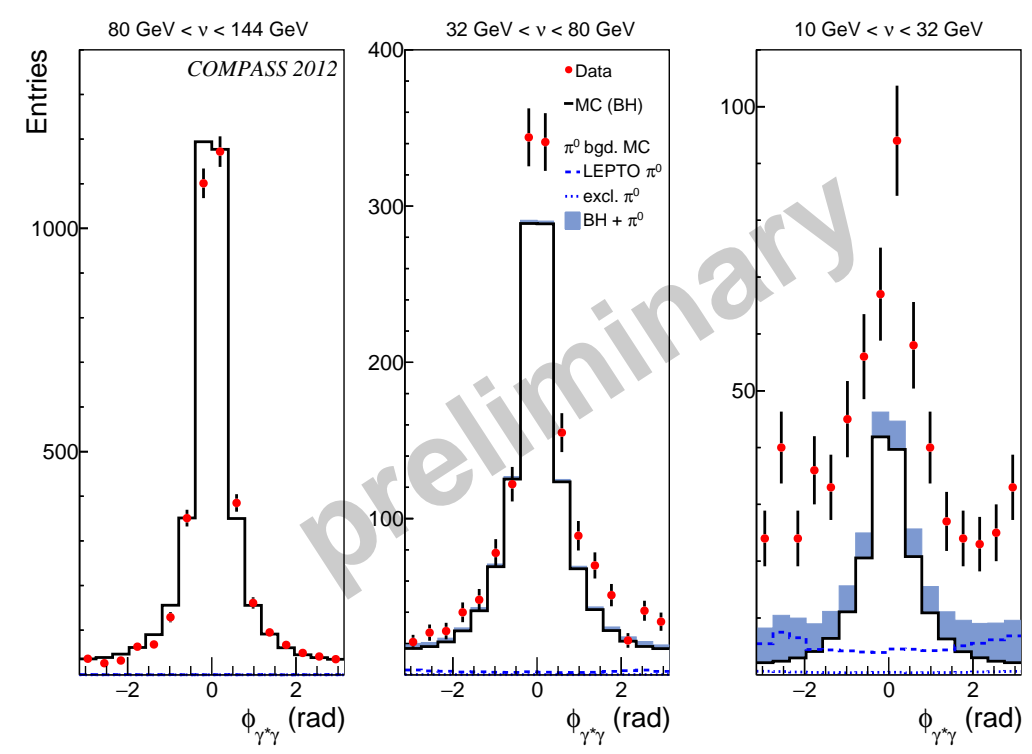

Figure 4: The exclusive single photon events obtained from the 2012 sample as a function of $\phi_{\gamma * \gamma}$ (identical to $\phi$ defined in the text) compared to the MC estimates of BH contribution (solid line) and the $\pi^{0}$ contamination (dotted and dashed lines). The Bethe-Heitler MC was normalised to the luminosity of 2012 data.

A way to identify the observed process, $\mu+p \rightarrow \mu^{\prime}+\gamma+p^{\prime}$, to which both the DVCS and Bethe-Heitler (BH) processes contribute, is to look at the angle $\phi$ between the lepton plane and the $\gamma^{*} \gamma$ plane. After applying all selections and for kinematic range $Q^{2}>1(\mathrm{GeV} / c)^{2}$ and $0.08<-t<$ $0.64(\mathrm{GeV} / c)^{2}$, where $t$ is the squared four-momentum transfer to the recoil proton, the observed $\phi$ distributions are displayed in Fig. 4 and compared to the predictions from the Monte Carlo (MC) simulations for the BH event yield and the $\pi^{0}$ contamination. The Bethe-Heitler MC is normalised to the luminosity of 2012 data. COMPASS offers the advantage to provide various kinematic domains, where either BH or DVCS dominates. The collection of almost pure BH events at large $\nu$ allows one to get an excellent reference yield and to control accurately the global efficiency of the apparatus. In contrast, the collection of a DVCS sample at smaller $\nu$ will allow the measurement of the $x_{\mathrm{Bj}}$ dependence of the $t$-slope of the cross section, which is related to the tomographic partonic image of the nucleon. In the intermediate domain, the DVCS contribution will be boosted by the $\mathrm{BH}$ process through the interference term that allows us to investigate DVCS at the amplitude level. The Bethe-Heitler contribution shows a characteristic peak at $\phi \simeq 0$. At the small $\nu$ there is a significant excess of events beyond the sum of the pure Bethe-Heitler and $\pi^{0}$ background contributions, which is represented as the shaded histogram. This excess is interpreted as an indication of DVCS events.

These pilot measurements allow us to extract the $t$-dependence of the pure DVCS cross section. The differential virtual-photon proton cross section $\mathrm{d} \sigma / \mathrm{d} t$ for this process is obtained in four bins of $t$ in the kinematic range of $10 \mathrm{GeV}<\nu<32 \mathrm{GeV}, 1(\mathrm{GeV} / \mathrm{c})^{2}<\mathrm{Q}^{2}<5(\mathrm{GeV} / \mathrm{c})^{2}$ and $0.08(\mathrm{GeV} / c)^{2}<-t<0.64(\mathrm{GeV} / c)^{2}$. The cross sections are evaluated first separately for the data taken with $\mu^{+}$and $\mu^{-}$beams, which have opposite polarisations. The extraction of the cross sections includes: normalisation to the luminosity for the corresponding data taking period, correction for $\pi^{0}$ background, correction for acceptance, subtraction of $\mathrm{BH}$ cross section and integration 


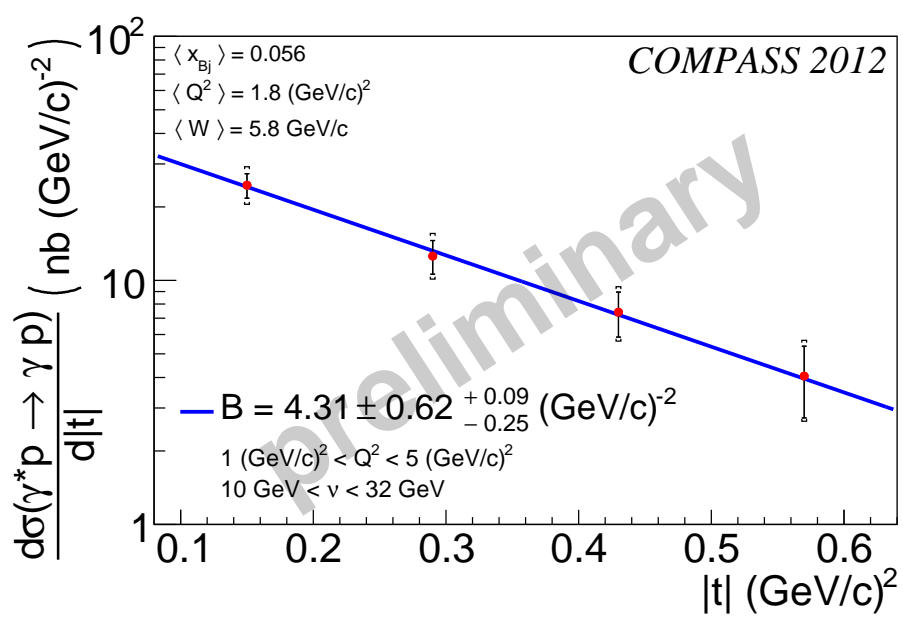

Figure 5: Differential DVCS cross section as a function of $t$. The inner error bars indicate the statistical uncertainties and the outer ones the combined statistical and systematic uncertainties.

over $\phi$ angle. The final cross section is evaluated as the mean of cross sections obtained with $\mu^{+}$ and $\mu^{-}$beams. This way all terms contributing to the single photon cross section cancel except the pure DVCS cross section.

The differential cross section for DVCS as a function of $t$ is shown in Fig. 5 together with the superimposed fitted function $\sim \exp (B t)$. The value of $t$-slope is $B=4.31 \pm 0.62_{-0.25}^{+0.09}(\mathrm{GeV} / \mathrm{c})^{-2}$, where the first error corresponds to the statistical uncertainty and the second one represents the (asymmetric) systematic uncertainty. As the slope $B$ is related to the transverse radius of parton distribution in the nucleon $r_{\perp}, B \simeq<r_{\perp}^{2}>$, this result provides the first information on the partonic transverse size of the nucleon in an uncharted up to now $x_{\mathrm{Bj}}$ range. It has an impact on the 'nucleon tomography', i.e. on the 3-D picture of the nucleon that correlates the transverse distibution of partons with their longitudinal momentum fraction $x_{\mathrm{Bj}}$. The $t$-slope parameter $B$ has been measured for DVCS at HERA at very small $x_{\mathrm{Bj}}$. The COMPASS result is compared to those from HERA in Fig. 6. In the $x_{\mathrm{Bj}}$ range covered by COMPASS the proton size is expected to shrink, which is consistent with the present measurements, albeit within still large uncertainties.

The projected accuracies for measurements of various observables within the GPD program are presented in the COMPASS-II proposal [14]. Investigation of GPDs with DVCS and HEMP on unpolarised protons using the data from 2016-2017 will allow to determine the $x_{\mathrm{Bj}}$-dependence for $t$-slopes of the differential cross sections that is related to the 'nucleon tomography'. Measurements of the beam charge and spin sum and difference of single- $\gamma$ cross sections will give access to the real and imaginary parts of the DVCS amplitude, and will allow to further constrain GPDs $H$. Studies of exclusive production of vector mesons $(\rho, \omega, \phi)$ will lead to the quark flavour and gluon separation for GPDs $H$, while that of exclusive $\pi^{0}$ production will provide constrains on the GPDs $\widetilde{E}$ and on chiral-odd GPDs $\bar{E}_{\mathrm{T}}[16]$.

The main goal of future measurements with transversely polarised target (possibly after 2020) is to constrain GPDs $E$, which are related to the orbital momentum of partons, and also to investigate the role of chiral-odd GPDs in exclusive meson production. 


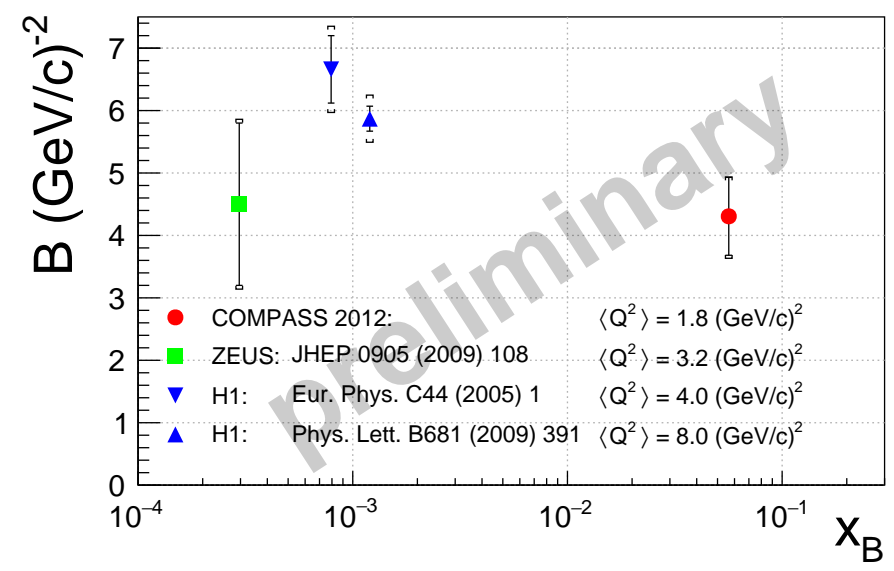

Figure 6: The slope $B$ of differential DVCS cross section measured at and COMPASS. The inner error bars indicate the statistical uncertainties and the outer ones the combined statistical and systematic uncertainties.

\section{References}

[1] D. Mueller et al, Fortsch. Phys. 42 (1994) 101.

[2] X. Ji, Phys. Rev. Lett. 78 (1997) 610; Phys. Rev. D 55 (1997) 7114.

[3] A.V. Radyushkin, Phys. Lett. B 385 (1996) 333; Phys. Rev. D 56 (1997) 5524.

[4] M. Burkardt, Phys. Rev. D 62 (2000) 071503; erratum-ibid. D 66 (2002) 119903; Int. J. Mod. Phys. A 18 (2003) 173; Phys. Lett. B 595 (2004) 245.

[5] K. Goeke, M.V. Polyakov and M. Vanderhaegen, Prog. Part. in Nucl. Phys. 47 (2001) 401.

[6] M. Diehl, Generalized Parton Distributions, DESY-thesis-2003-018, hep-ph/0307382.

[7] A.V. Belitsky and A.V.Radyushkin, Phys. Rep. 418 (2005) 1.

[8] COMPASS Collaboration, P. Abbon et al., Nucl. Instr. Meth. A 577 (2007) 455.

[9] C. Adolph et al. [COMPASS Collaboration], Nucl. Phys. B 865 (2012) 1.

[10] C. Adolph et al. [COMPASS Collaboration], Phys. Lett. B 731 (2014) 19.

[11] S.V. Goloskokov and P. Kroll, Eur. Phys. J. C 74 (2014) 2725;

S.V. Goloskokov and P. Kroll, private communication.

[12] S.V. Goloskokov and P. Kroll, Eur. Phys. J. A 50 (2014) 146;

S.V. Goloskokov and P. Kroll, private communication.

[13] A. Airapetian et al. [HERMES Collaboration], Eur. Phys. J. C 75 (2015) 600.

[14] F. Gautheron et al. [COMPASS Collaboration], CERN-SPSC-2010-014, SPSC-P-340, May 2010.

[15] Ph. Joerg, The t-dependence of the pure DVCS cross-section at COMPASS, DIS-2016 Workshop, DESY, Hamburg, https://indico.desy.de/contributionDisplay.py?sessionId=6\&contribId=198\& confId=12482

[16] S.V. Goloskokov and P. Kroll, Eur. Phys. J. A 47 (2011) 112. 